\title{
Kinematic and compliance analysis for tendon-driven robotic mechanisms with flexible tendons
}

\author{
Sun-Lai Chang ${ }^{a}$, Jyh-Jone Lee ${ }^{\mathrm{b}, *}$, Home-Che Yen ${ }^{\mathrm{b}}$ \\ a Service Apogee, Inc., Plano, TX, USA \\ ${ }^{\mathrm{b}}$ Department of Mechanical Engineering, National Taiwan University, Taipei, Taiwan 106, ROC
}

Received 24 May 2004; received in revised form 16 November 2004; accepted 23 November 2004

Available online 14 March 2005

\begin{abstract}
Compliance in tendon transmission may result in position errors for tendon-driven robotic mechanisms. As a consequence, the study of compliance of the mechanism is important for control purpose. This paper deals with the kinematic and force analysis of tendon-driven robotic mechanisms with compliant tendons. The displacement equation of tendon-driven robotic mechanisms that takes tendon compliance into account can be systematically determined from the kinematic structure. The force relations between the joint space and the tendon space are investigated. In particular, the equations relating joint torques, joint angles and tendon displacements are derived. The compliance of the mechanism is thereafter established. Dynamic equations of the system are also analyzed and system's resonant frequencies are derived through local linearization of the dynamic model. The proposed analysis can be useful for a fast evaluation for the static and dynamic performance of tendon-driven robotic mechanisms.
\end{abstract}

(C) 2005 Elsevier Ltd. All rights reserved.

Keywords: Tendon-driven mechanisms; Fundamental circuit; Compliance; Resonant frequency

\footnotetext{
${ }^{*}$ Corresponding author. Tel.: +88622364 8501; fax: +886223631755.

E-mail address: jjlee@ccms.ntu.edu.tw (J.-J. Lee).
} 


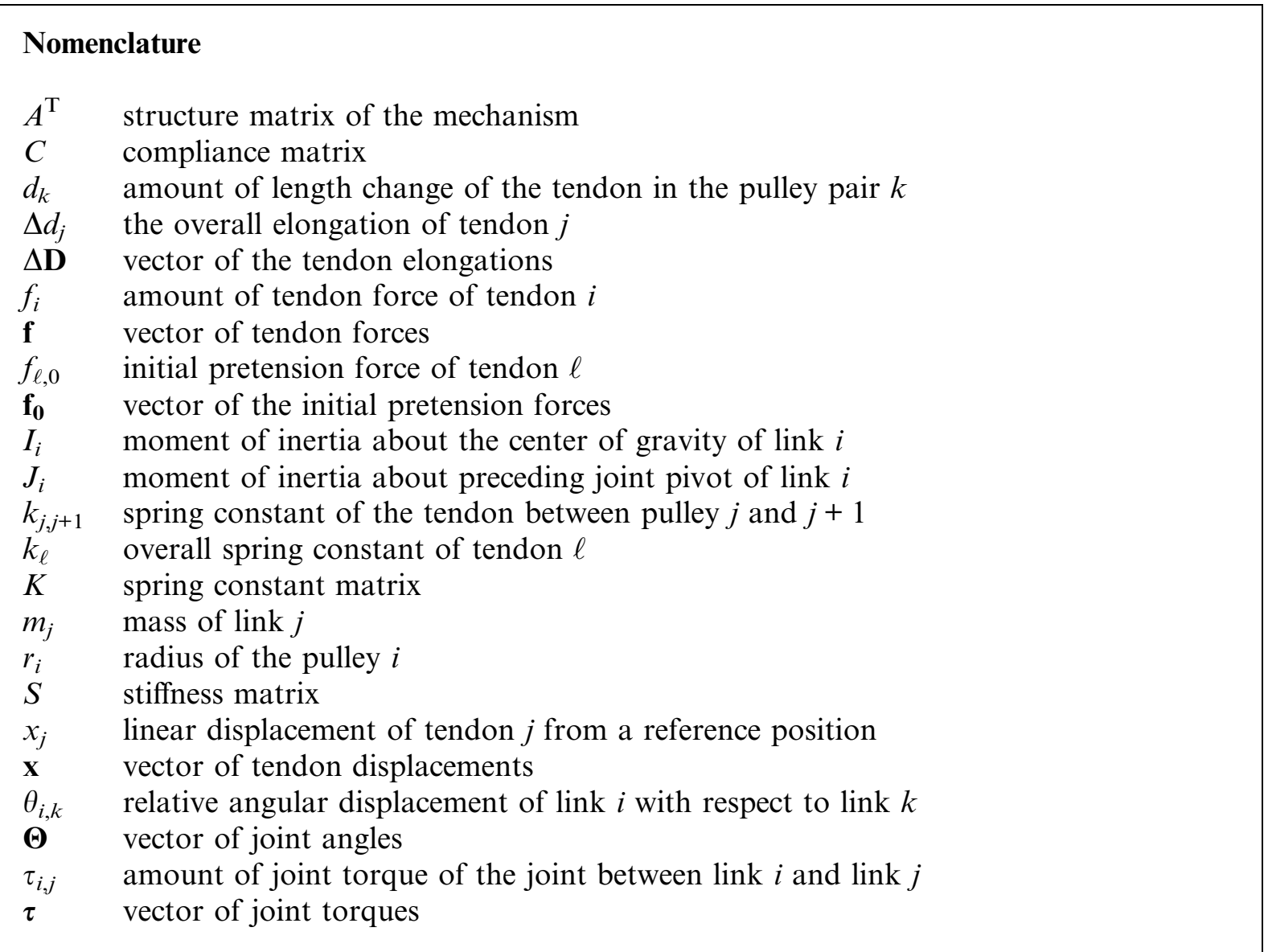

\section{Introduction}

In the past decades, robotic manipulators using tendon transmission have been enthusiastically studied in a number of areas such as dexterous hands [1,2], parallel cable-suspended manipulators $[3,4]$, teleoperating robots [5] and robots for biological use [6]. The major advantage of using tendon transmission lies in that actuators can be installed on the remote base such that a lightweight and compact design can be realized. However, using tendon transmission may introduce other problems such as friction and compliance in tendons and/or increasing extra components used in the system. These may increase the complexity for the control of the system. In reality, tendon-driven robotic manipulators are more compliant than geared robotic and direct-driven robotic manipulators because tendons are more flexible than those components. This property usually degrades accuracy in positioning, lowers response speed, and shifts eigenfrequencies to lower levels. Therefore, it would be essential to research on the transmission compliance as well as dynamic characteristics for fast and accurate motion control of such systems. To realize the nature of tendon-driven robotic manipulators, a great number of analytical methods for such systems have been proposed and studied. The kinematics and statics of articulated tendon-driven robotic mechanisms 
were investigated by Morecki et al. [7], Salisbury [1], and Tsai and Lee [8]. Ideal tendons with no compliance were assumed in their studies. Hollars and Cannon [9] experimented on the control of a two-link manipulator with flexible tendons. They concluded that compliance in tendon drives had a significant effect on the system control. Prisco and Bergamasco [10] derived the dynamics of a type $(2 \mathrm{~N})$ of multi-degree-of-freedom (DOF) tendon-driven manipulators using the Lagrangian method. Lee and Lee [11] proposed a new model for the tendon tension and performed the dynamic analysis. On the other hand, there is also some literature investigating on the performance of single-DOF tendon devices $[12,13]$ of which the kinematic structure is less coupled than that of the multi-DOF system. Except for these, not much literature dealing with the kinematic and compliance analysis of multi-DOF systems with flexible tendons can be found. Thus, the objective of this paper is to establish a systematic procedure for the kinematic and compliance analysis of multiDOF, tendon-driven robotic mechanisms. This paper is organized as follows. First, a systematic methodology for kinematic analysis of tendon-driven manipulators with compliant tendons is presented. Next the force relations between the joint space and the tendon space are investigated. Compliance of the system is subsequently determined. Then, the dynamic equations associated with the resonant frequencies and mode shapes of the system are also derived. Finally, a twoDOF tendon-driven manipulator is demonstrated for the generalization of procedure.

\section{Assumptions}

To adapt to the scope of this work, the following assumptions for the tendon-driven robotic manipulator are considered.

(I) The robotic manipulator is of articulated type, i.e., after the removal of tendons and intermediate pulleys, the manipulator becomes a serial type.

(II) All tendons are under tension. The amount of stretch in tendon is small and linearly proportional to the tendon force.

(III) No slippage occurs between pulleys and tendons.

(IV) Tendons are lightweight such that the weight, flexural bending and shear effects of tendons will not be considered.

\section{Kinematics}

The theory of fundamental circuits and the coaxially conditions have been applied to the kinematic analysis of geared mechanisms as well as tendon-driven mechanisms with ideally rigid tendons $[8,14]$. In this section, we will show that the concept is still applicable to the analysis of tendon-driven robotic mechanisms with non-rigid tendons.

\subsection{Fundamental circuit equations}

Fig. 1 shows the planar schematic of a simple tendon-and-pulley train, where $i, j$ are the two pulleys and $k$ serves as the common carrier. Pulley $i$ and $j$ are coupled by a flexible tendon having a spring constant. For the purpose of analysis, the counter-clockwise direction is temporarily 


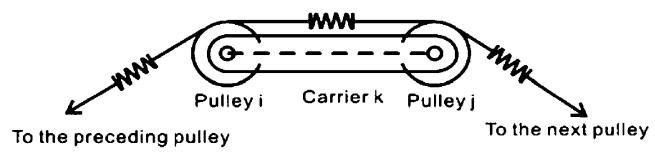

Fig. 1. A parallel routing pulley pair with flexible tendon.

assigned as the positive direction of rotation for each pulley. It is obvious that the relative angular displacements of the two pulleys with respect to the carrier will be differentiated by a small amount of tendon stretch. Therefore, a revised fundamental circuit equation relating the rotations of the two pulleys can be written as

$$
r_{i} \theta_{i, k}= \pm r_{j} \theta_{j, k}+d_{k}
$$

where $\theta_{i, k}$ and $\theta_{j, k}$ respectively denote relative angular displacements of pulleys $i$ and $j$ with respect to the carrier $k, r_{i}$ and $r_{j}$ denote the radii of the two pulleys, and $d_{k}$ the amount of length change of the tendon connecting the two pulleys with respect to the equilibrium state. The \pm sign is to be determined by the tendon routing topology. The sign is positive for a parallel routing (as Fig. 1 shows) and negative for a crossed routing.

\subsection{Coaxiality}

Suppose three links $i, j$, and $k$ share a common joint axis. Then, the relative motion among these links can be related by the chain rule:

$$
\theta_{i, k}=\theta_{i, j}+\theta_{j, k}
$$

\subsection{Transmission lines}

Fig. 2 shows a typical transmission line in which the tendon is routed from one of the moving links over pulleys mounted on the joint axes to the base link. For convenience, the links are

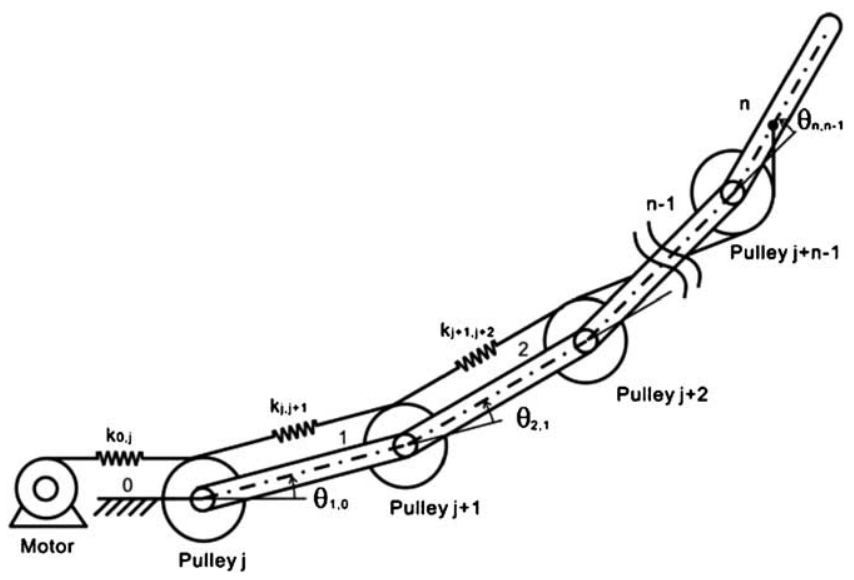

Fig. 2. A transmission line with flexible tendon. 
labeled sequentially from 0 to $n$ and the pulleys are labeled from $j$ to $j+n-1$. Then, the fundamental circuit equation can be written once for each pulley pair as

$$
r_{j+i-1} \theta_{j+i-1, i}= \pm r_{j+i} \theta_{j+i, i}+d_{i} \text { for } i=1,2, \ldots, n-1
$$

Since link $i-1, i$ and pulley $j+i-1$ are coaxial, the coaxiality relation can be applied as

$$
\theta_{j+i-1, i}=\theta_{j+i-1, i-1}-\theta_{i, i-1} \quad \text { for } i=1,2, \ldots, n
$$

Note the last (distal) pulley $j+n-1$ is fixed to link $n, \theta_{j+n-1, n}=0$. Suppressing $\theta_{j+i-1, i}$ for $i=2,3, \ldots, n$ from Eqs. (3) and (4), yields

$$
\begin{aligned}
\theta_{j, 0}= & \theta_{1,0} \pm\left(r_{j+1} / r_{j}\right) \theta_{2,1} \pm\left(r_{j+2} / r_{j}\right) \theta_{3,2} \pm \cdots \pm\left(r_{j+n-1} / r_{j}\right) \theta_{n, n-1}+\left(1 / r_{j}\right) d_{1} \pm\left(1 / r_{j}\right) d_{2} \\
& \pm \cdots \pm\left(1 / r_{j}\right) d_{n-1}
\end{aligned}
$$

If the structure is in equilibrium state, then Eq. (5a) can be rewritten as

$$
\theta_{j, 0}=\theta_{1,0} \pm\left(r_{j+1} / r_{j}\right) \theta_{2,1} \pm\left(r_{j+2} / r_{j}\right) \theta_{3,2} \pm \cdots \pm\left(r_{j+n-1} / r_{j}\right) \theta_{n, n-1}+\left(1 / r_{j}\right) \Delta d_{j}
$$

where $\Delta d_{j}=d_{1} \pm d_{2} \pm \cdots \pm d_{n-1}$ is the overall elongation of tendon $j$. The \pm sign for each term of $\theta_{k, k-1}$ above is determined by the number of crossed routings preceding the $k$ th joint axis. The sign is positive if the number of crossed routing is even; otherwise it is negative. Let the linear displacement of tendon $j$ from a reference position be $x_{j}$. The displacement relation between the $x_{j}$ and base pulley $\theta_{j, 0}$ can be written as

$$
x_{j}= \pm r_{j} \theta_{j, 0}
$$

where the \pm sign is positive provided that a pull of the tendon causes a positive rotation of the base pulley, otherwise it is negative. By substituting Eq. (5b) into (6), for each transmission line, and assembling each equation into matrix form, we obtain

$$
\mathbf{x}=A \boldsymbol{\Theta}+\Delta \mathbf{D}
$$

where $\mathbf{x}=\left[x_{1}, x_{2}, \ldots, x_{m}\right]^{\mathrm{T}}$ denotes $m$-dimensional vector of tendon displacements, $\boldsymbol{\Theta}=\left[\theta_{1,0}, \theta_{2,1}\right.$, $\left.\ldots, \theta_{n, n-1}\right]^{\mathrm{T}}$ denotes an $n$-dimensional vector of the joint angles, $\Delta \mathbf{D}=\left[\Delta d_{1}, \Delta d_{2}, \ldots, \Delta d_{m}\right]^{\mathrm{T}}$ denotes an $m$-dimensional vector of the tendon elongations and $A$ is an $m \times n$ matrix whose elements are functions of the pulley sizes and the tendon routing topology. From Eq. (7), it can be seen that the linear displacements of tendons are given by two terms as the non-elongated displacement due to joint rotations and the displacement due to tendon elongations. The latter term contributes to the difference between a non-flexible tendon drive and a flexible tendon drive.

\section{Forces and compliance}

It has been shown that for ideally non-stretchable tendons the tendon force, $\mathbf{f}$, exerted by the tendons are related to the joint torques, $\tau$, by the following relationship [14]:

$$
\tau=A^{\mathrm{T}} \mathbf{f}
$$

where $\tau$ is denoted by $\left[\tau_{1,0}, \tau_{2,1}, \ldots, \tau_{n, n-1}\right]^{\mathrm{T}}$, f is denoted by $\left[f_{1}, f_{2}, \ldots, f_{m}\right]^{\mathrm{T}}$, and $A^{\mathrm{T}}$ is called the structure matrix. Lee and Lee [11] further demonstrated that the relationship still remains valid 
for the model with flexible tendons as well as that each segment on one tendon is of equal tension, provided that the mass/inertias of the intermediate pulleys are neglected.

When a force is applied upon a manipulator, the tendons in the system are deflected. The manipulator's structural compliance may result from the combined effects of transmission compliance, link compliance, and compliance of the environment that the robot acts on. The stiffness of tendon can be related to the tendon forces by using Hooke's law. Noting that $k_{j, j+1}$ is the spring constant for each segment of tendon connecting pulley $j$ and $j+1$ shown in Fig. 2, the tendon force equation can be written once for each tendon as

$$
f_{\ell}=f_{\ell, 0}+k_{\ell} \Delta d_{\ell} \quad \ell=1,2, \ldots, m
$$

where $k_{\ell}=1 /\left(1 / k_{0, j}+1 / k_{j, j+1}+\ldots+1 / k_{j+n-2, j+n-1}\right), f_{\ell, 0}$, and $\Delta d_{\ell}$ are respectively the overall spring constant, the initial pretension, and the elongation of the $\ell$ th tendon. Assembling these $m$ equations in matrix form yields

$$
\mathbf{f}=\mathbf{f}_{\mathbf{0}}+K \Delta \mathbf{D}
$$

where $\mathbf{f}_{\mathbf{0}}=\left[f_{1,0}, f_{2,0}, \ldots, f_{m, 0}\right]^{\mathrm{T}}$ denotes the vector of initial pretension of tendons, and $K$ denotes an $m \times m$ diagonal matrix whose elements are the spring constants of tendons. Substituting Eq. (7) into (10) to eliminate $\Delta \mathbf{D}$, and then into Eq. (8), we obtain

$$
\tau=A^{\mathrm{T}} K \mathbf{x}-A^{\mathrm{T}} K A \boldsymbol{\Theta}
$$

More specifically, for the case of the number of tendon exceeding the number of degrees of freedom, $m>n$ and $\operatorname{rank}(A)=n$, once $\mathbf{x}$ and $\tau$ are given, the joint angles can be obtained as

$$
\boldsymbol{\Theta}=\left(A^{\mathrm{T}} K A\right)^{-1} A^{\mathrm{T}} K \mathbf{x}-\left(A^{\mathrm{T}} K A\right)^{-1} \boldsymbol{\tau}
$$

On the other hand, given joint angles $\Theta$ and joint torques $\tau$, the linear displacements of tendons is given by

$$
\mathbf{x}=A \boldsymbol{\Theta}+\left(A^{\mathrm{T}} K\right)^{+} \tau+N \boldsymbol{\lambda}
$$

where $\left(A^{\mathrm{T}} K\right)^{+}=\left(A^{\mathrm{T}} K\right)^{\mathrm{T}}\left[\left(A^{\mathrm{T}} K\right)\left(A^{\mathrm{T}} K\right)^{\mathrm{T}}\right]^{-1}$ is the pseudo-inverse of $\left(A^{\mathrm{T}} K\right), N$ is an $m \times(m-n)$ matrix with its column vectors spanning the null space of $\left(A^{\mathrm{T}} K\right)$, and $\lambda$ is an arbitrary $(m-n)$ dimensional vector. Note that the first term on the right-hand side of Eq. (11c) is the value contributed by joint angles, the second term denotes the value contributed by joint torques, and the third term gives the ratios of tendon displacements that result in neither net joint torques nor joint angles.

\subsection{Structure compliance}

In case the mechanism is being held stationary, for example, at certain posture along the planned trajectory, the actuators need to be held still and the displacements of tendons remain fixed. Then, from Eq. (11a) the joint torques perturbed due to a small variation of joint angles can be derived as

$$
\delta \tau=-A^{\mathrm{T}} K A \delta \Theta=-S \delta \Theta
$$


or

$$
\delta \Theta=-C \delta \tau
$$

where $S=\left(A^{\mathrm{T}} K A\right)$ denotes the stiffness matrix and $C=S^{-1}$ denotes the compliance matrix of the system. The errors of the joint angles caused by the variation of joint torques can thus be calculated from Eq. (12b).

\section{Dynamics and resonant frequency}

The dynamics of a robot system is usually a very complicated subject. The dynamic characteristics, such as frequency responses and mode shapes, are non-linear, configuration-dependent, and complex to solve. However, for some applications, such as path planning for certain tasks, it is necessary to rapidly move the end effector of a robot along the desired trajectory. By examining the dynamic characteristics of the model, it is possible to predict how a robotic system will behave. Thus, fast calculation, which is usually performed by the local linearization along its trajectory, of the dynamic model is important for real-time control of the robot system. The dynamic equations of an articulated robotic system can be written as

$$
\ddot{\boldsymbol{\Theta}}=G(\dot{\boldsymbol{\Theta}}, \boldsymbol{\Theta}, \tau)
$$

where $\boldsymbol{\Theta}$ is the $n \times 1$ vector of joint angles. Representing the dynamic equations in state-variable form yields

$$
\dot{\mathbf{y}}=F(\mathbf{y})+H(\mathbf{y})
$$

where $\mathbf{y}=\left[y_{1}, y_{2}, \ldots, y_{2 n}\right]^{\mathrm{T}}$ is a $2 n \times 1$ state vector whose elements are defined as

$$
\left[y_{2 j-1}, y_{2 j}\right]=\left[\theta_{j}, \dot{\theta}_{j}\right] \quad(j=1,2, \ldots, n)
$$

and $F$ and $H$ are $2 n \times 1$ and $2 n \times n$ matrices, respectively. Note that $\boldsymbol{\Theta}$ and $\mathbf{y}$ can be further expressed as

$$
\boldsymbol{\Theta}=U \mathbf{y}
$$

where $U=\left[u_{i j}\right]$ is an $n \times 2 n$ matrix whose elements are given by

$$
u_{i j}= \begin{cases}1 & \text { for } j=2 i-1 \\ 0 & \text { otherwise }\end{cases}
$$

Substituting Eqs. (11a) and (15) into (14), yields

$$
\dot{\mathbf{y}}=F(\mathbf{y})-H(\mathbf{y}) A^{\mathrm{T}} K A U \mathbf{y}+H(\mathbf{y}) A^{\mathrm{T}} K \mathbf{x}
$$

Now consider the system is operated at the vicinity of a specific position where the tendon displacements $\mathbf{x}=\mathbf{x}^{*}$ and joint angles $\boldsymbol{\Theta}=\boldsymbol{\Theta}^{*}$. The variations of tendon elongation with respect to the specific position can be neglected due to the small scale. Thus, writing Eq. (7) at this specific position gives

$$
\mathbf{x}^{*}=A U \mathbf{y}^{*}
$$


Also, the state can be changed from $\mathbf{y}$ to the new equilibrium point of this system as [15]

$$
\mathbf{z}=\mathbf{y}-\mathbf{y}^{*}
$$

By substituting Eqs. (17a) and (17b) into (16), we have

$$
\dot{\mathbf{z}}=F\left(\mathbf{z}+\mathbf{y}^{*}\right)-H\left(\mathbf{z}+\mathbf{y}^{*}\right) A^{\mathrm{T}} K A U \mathbf{z}
$$

The above equation can be further linearized as

$$
\dot{\mathbf{Z}}=L \mathbf{z}
$$

where

$$
L=\partial\left[F\left(\mathbf{z}+\mathbf{y}^{*}\right)-H\left(\mathbf{z}+\mathbf{y}^{*}\right) A^{\mathrm{T}} K A U \mathbf{z}\right]_{\mathbf{z}=0} / \partial \mathbf{z}
$$

or

$$
L=\partial F\left(\mathbf{y}^{*}\right) / \partial y-H\left(\mathbf{y}^{*}\right) A^{\mathrm{T}} K A U
$$

The resonant frequencies and mode shapes can be obtained by calculating the eigenvalues and eigenvectors of the linearized system matrix $L$. These values can thus provide us a fast estimate of the dynamic behaviors of the system.

\subsection{Example}

To illustrate the generalization of the principle, a two-DOF tendon-driven device is used to demonstrate the process. Fig. 3 shows the two-jointed, four link robotic mechanism driven by three open-ended tendons. The tendons are spooled to the actuators located at the base link 0 . In this system, each tendon is flexible and a certain spring constant is assumed for each segment of tendon between two intermediate pulleys. The dynamic equations for the mechanism have been derived in [11] and are listed in appendix for convenience. If the moment of inertias of pulleys 3 and 4 are neglected, the dynamic equations of the system can be reduced to

$$
\begin{aligned}
& -r_{3} f_{1}-r_{4} f_{2}+r_{1} f_{3} \\
& =\tau_{1,0}=I_{1} \ddot{\theta}_{1,0}+I_{2}\left(\ddot{\theta}_{1,0}+\ddot{\theta}_{2,1}\right)-a_{1}^{2}\left(m_{1}+m_{2}\right) \ddot{\theta}_{1,0}-m_{1} b_{1}^{2} \ddot{\theta}_{1,0}-m_{2}\left(a_{2}-b_{2}\right) \\
& \quad \times\left[a_{1}\left(\operatorname{Sin} \theta_{2,1} \dot{\theta}_{1,0}^{2}+\operatorname{Cos} \theta_{2,1} \ddot{\theta}_{1,0}\right)+\left(a_{2}-b_{2}\right)\left(\ddot{\theta}_{1,0}+\ddot{\theta}_{2,1}\right)\right]+a_{1}\left\{2 m_{1} b_{1} \ddot{\theta}_{1,0}+m_{2}\left(a_{2}-b_{2}\right)\right. \\
& \left.\quad \times\left[\operatorname{Sin} \theta_{2,1}\left(\dot{\theta}_{1,0}+\dot{\theta}_{2,1}\right)^{2}-\operatorname{Cos} \theta_{2,1}\left(\ddot{\theta}_{1,0}+\ddot{\theta}_{2,1}\right)\right]\right\} \\
& r_{2} f_{1}-r_{2} f_{2}=\tau_{2,1} \\
& =m_{2}\left(a_{2}-b_{2}\right)\left[a_{1}\left(\operatorname{Sin} \theta_{2,1} \dot{\theta}_{1,0}^{2}+\operatorname{Cos} \theta_{2,1} \ddot{\theta}_{1,0}\right)+\left(a_{2}-b_{2}\right)\left(\ddot{\theta}_{1,0}+\ddot{\theta}_{2,1}\right)\right]+I_{2}\left(\ddot{\theta}_{1,0}+\ddot{\theta}_{2,1}\right)
\end{aligned}
$$

where $I_{1}$ and $I_{2}$ are the moment of inertia about the center of gravity, and $m_{1}$ and $m_{2}$ are the masses of link 1 and 2. Arranging the above equations in matrix form yields

$$
A^{\mathrm{T}} \mathbf{f}=\tau
$$

where $\mathbf{f}=\left[f_{1}, f_{2}, f_{3}\right]^{\mathrm{T}}$ and $A^{\mathrm{T}}=\left[\begin{array}{ccc}-r_{3} & -r_{4} & r_{1} \\ r_{2} & -r_{2} & 0\end{array}\right]$. 


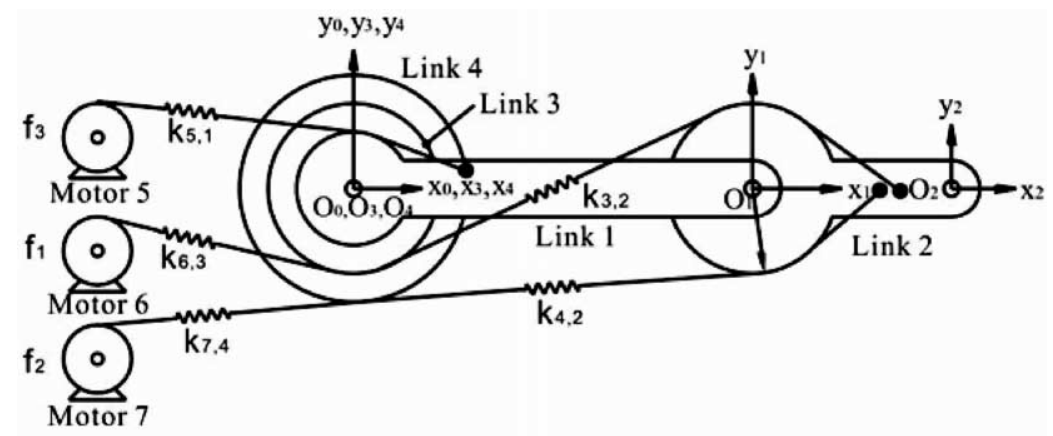

(a)

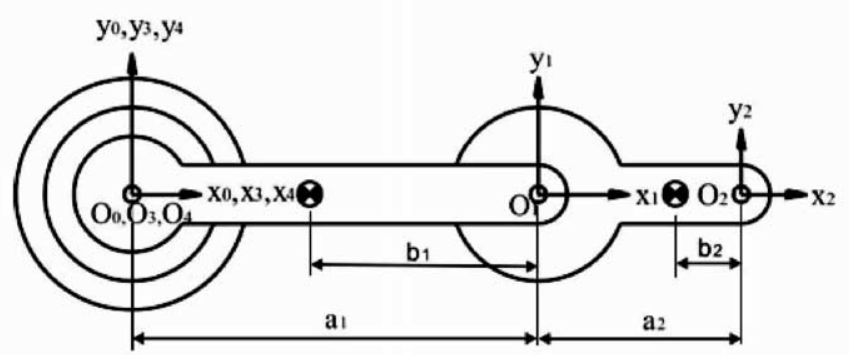

(b)

Fig. 3. (a) A robotic manipulator driven by flexible tendons and (b) kinematics of (a).

Thus, the stiffness of the system is given by Eq. (12a)

$$
S=\left(A^{\mathrm{T}} K A\right)=\left[\begin{array}{cc}
r_{3}^{2} k_{1}+r_{4}^{2} k_{2}+r_{1}^{2} k_{3} & -r_{2} r_{3} k_{1}+r_{2} r_{4} k_{2} \\
-r_{2} r_{3} k_{1}+r_{2} r_{4} k_{2} & r_{2}^{2} k_{1}+r_{2}^{2} k_{2}
\end{array}\right]
$$

while the compliance $C$ is the inverse of $S$.

\subsection{Resonant frequencies}

Representing the dynamic equations in state-space form, yields

$$
\dot{\mathbf{y}}=F(\mathbf{y})+H(\mathbf{y}) \tau
$$

where $\mathbf{y}=\left[y_{1}, y_{2}, y_{3}, y_{4}\right]^{\mathrm{T}}=\left[\theta_{1,0}, \dot{\theta}_{1,0}, \theta_{2,1}, \dot{\theta}_{2,1}\right]^{\mathrm{T}}, \boldsymbol{\tau}=\left[\tau_{1,0}, \tau_{2,1}\right]^{\mathrm{T}}$,

$$
F(y)=\left[\begin{array}{c}
y_{2} \\
p_{1} y_{2}^{2}+p_{2} y_{4}^{2}+p_{3} y_{2} y_{4} \\
y_{4} \\
q_{1} y_{2}^{2}+q_{2} y_{4}^{2}+q_{3} y_{2} y_{4}
\end{array}\right]
$$




$$
H(y)=\left[\begin{array}{cc}
0 & 0 \\
p_{4} & p_{5} \\
0 & 0 \\
q_{4} & q_{5}
\end{array}\right]
$$

and where

$$
\begin{aligned}
& p_{1}=\left[e_{1}\left(J_{2}+e_{1} \operatorname{Cos} \theta_{2,1}\right) \operatorname{Sin} \theta_{2,1}\right] / e_{2}, \\
& p_{2}=\left(e_{1} J_{2} \operatorname{Sin} \theta_{2,1}\right) / e_{2}, \\
& p_{3}=\left(2 e_{1} J_{2} \operatorname{Sin} \theta_{2,1}\right) / e_{2}, \\
& p_{4}=J_{2} / e_{2}, \\
& p_{5}=-\left(J_{2}+e_{1} \operatorname{Cos} \theta_{2,1}\right) / e_{2}, \\
& q_{1}=-\left[e_{1}\left(J_{1}+J_{2}+2 e_{1} \operatorname{Cos} \theta_{2,1}+m_{2} a_{1}^{2}\right) \operatorname{Sin} \theta_{2,1}\right] / e_{2}, \\
& q_{2}=-\left[e_{1}\left(J_{2}+e_{1} \operatorname{Cos} \theta_{2,1}\right) \operatorname{Sin} \theta_{2,1}\right] / e_{2}, \\
& q_{3}=-\left[2 e_{1}\left(J_{2}+e_{1} \operatorname{Cos} \theta_{2,1}\right) \operatorname{Sin} \theta_{2,1}\right] / e_{2}, \\
& q_{4}=-\left(J_{2}+e_{1} \operatorname{Cos} \theta_{2,1}\right) / e_{2},
\end{aligned}
$$

and $q_{5}=\left(J_{1}+J_{2}+2 e_{1} \operatorname{Cos} \theta_{2,1}+m_{2} a_{1}^{2}\right) / e_{2}$, and where $e_{1}=m_{2} a_{1}\left(a_{2}-b_{2}\right), e_{2}=J_{1} J_{2}-n^{2} \operatorname{Cos}^{2} \theta_{2,1}+$ $J_{2} m_{2} a_{1}^{2}$, and $J_{1}, J_{2}$ are respectively the moment of inertia about its preceding joint pivot of links 1 and 2. Upon substitution of Eqs. (21b) and (21c) into (19b), we obtain

$$
\begin{aligned}
L= & {\left[\begin{array}{cccc}
0 & 1 & 0 & 0 \\
0 & 2 p_{1} y_{2}+p_{3} y_{4} & y_{2}^{2} \frac{\partial p_{1}}{\partial \theta_{2,1}}+y_{4}^{2} \frac{\partial p_{2}}{\partial \theta_{2,1}}+y_{2} y_{4} \frac{\partial p_{3}}{\partial \theta_{2,1}} & 2 p_{2} y_{4}+p_{3} y_{2} \\
0 & 0 & 0 & 1 \\
0 & 2 q_{1} y_{2}+q_{3} y_{4} & y_{2}^{2} \frac{\partial q_{1}}{\partial \theta_{2,1}}+y_{4}^{2} \frac{\partial q_{2}}{\partial \theta_{2,1}}+y_{2} y_{4} \frac{\partial q_{3}}{\partial \theta_{2,1}} & 2 q_{2} y_{4}+q_{3} y_{2}
\end{array}\right] } \\
& -\left[\begin{array}{cccc}
0 & 0 & 0 & 0 \\
p_{4} s_{11}+p_{5} s_{21} & 0 & p_{4} s_{12}+p_{5} s_{22} & 0 \\
0 & 0 & 0 & 0 \\
q_{4} s_{11}+q_{5} s_{21} & 0 & q_{4} s_{12}+q_{5} s_{22} & 0
\end{array}\right]
\end{aligned}
$$

where $s_{11}=k_{2} r_{4}^{2}+k_{1} r_{3}^{2}+k_{3} r_{1}^{2}, s_{12}=k_{2} r_{4} r_{2}-k_{1} r_{3} r_{2}, s_{21}=s_{12}$, and $s_{22}=k_{2} r_{2}^{2}+k_{1} r_{2}^{2}$. By letting $y_{1}=\theta_{1,0}, y_{2}=\dot{\theta}_{1,0}=0, y_{3}=\theta_{2,1}$, and $y_{4}=\dot{\theta}_{2,1}=0$ for the equilibrium position, the equations of motion can be reduced to

$$
\left[\begin{array}{l}
\ddot{\theta}_{1,0} \\
\ddot{\theta}_{2,1}
\end{array}\right]=-\left[\begin{array}{ll}
p_{4} s_{11}+p_{5} s_{21} & p_{4} s_{12}+p_{5} s_{22} \\
q_{4} s_{11}+q_{5} s_{21} & q_{4} s_{12}+q_{5} s_{22}
\end{array}\right]\left[\begin{array}{c}
\theta_{1,0} \\
\theta_{2,1}
\end{array}\right]
$$

Thus, the square roots of the eigenvalues of the matrix are the resonant frequencies of this system, and its corresponding eigenvectors are the mode shapes of the structure. 


\section{Conclusion}

A systematic methodology for the kinematic and compliance analysis of tendon-driven robotic manipulator with compliant tendons has been developed. The displacement equation of tendons is established via the concept of fundamental circuit. A relationship between joint torque and tendon elongation are established and the compliance of the tendon-driven system is derived. Dynamic equations associated with the resonant frequencies and mode shapes of the system are investigated through local linearization of the equations of motion. An example is presented to illustrate the procedure of obtaining the compliance and resonant frequencies. The results presented in this work are helpful in the expeditious evaluation of the compliance as well as dynamic characteristics of tendon-driven robotic systems with flexible tendons.

\section{Appendix A}

For the four-link system shown in Fig. 3, the equations of motion are

$$
\begin{aligned}
& I_{2}\left(\ddot{\theta}_{1,0}+\ddot{\theta}_{2,1}\right)=-m_{2}\left(a_{2}-b_{2}\right)\left[a_{1}\left(\operatorname{Sin} \theta_{2,1} \dot{\theta}_{1,0}^{2}+\operatorname{Cos} \theta_{2,1} \ddot{\theta}_{1,0}\right)+\left(a_{2}-b_{2}\right)\left(\ddot{\theta}_{1,0}+\ddot{\theta}_{2,1}\right)\right] \\
& \quad+r_{2}\left(f_{2,3}-f_{2,4}\right) \\
& I_{3} \ddot{\theta}_{3,0}=r_{3}\left(f_{3,2}-f_{1}\right) \\
& I_{4} \ddot{\theta}_{4,0}=r_{4}\left(f_{4,2}-f_{2}\right) \\
& \ddot{I}_{1} \ddot{\theta}_{1,0}=-\left(r_{2}+r_{3}\right) f_{2,3}+\left(r_{2}-r_{4}\right) f_{2,4}+r_{1} f_{3}-a_{1}^{2}\left(m_{1}+m_{2}\right) \ddot{\theta}_{1,0}-m_{1} b_{1}^{2} \ddot{\theta}_{1,0} \\
& \quad+a_{1}\left\{2 m_{1} b_{1} \ddot{\theta}_{1,0}+m_{2}\left(a_{2}-b_{2}\right)\left[\operatorname{Sin} \theta_{2,1}\left(\dot{\theta}_{1,0}+\dot{\theta}_{2,1}\right)^{2}-\operatorname{Cos} \theta_{2,1}\left(\ddot{\theta}_{1,0}+\ddot{\theta}_{2,1}\right)\right]\right\}
\end{aligned}
$$

where $f_{i, j}$ is the tendon tension acting on link $i$ by link $j$.

\section{References}

[1] J.K. Salisbury, Kinematic and force analysis of articulated hands, Ph.D. Dissertation, Department of Mechanical Engineering, Stanford University, Stanford, CA, 1982.

[2] S.C. Jacobsen, J.E. Wood, D.F. Knutti, K.B. Biggers, The Utah-MIT dexterous hand: work in progress, Int. J. Rob. Res. 3 (4) (1985) 21-50.

[3] J. Albus, R. Bostelman, N. Dagalakis, The NIST spider, a robot crane, J. Res. NIST 97 (3) (1992) $373-385$.

[4] S. Kawamura, W. Choe, S. Tanaka, S.R. Pandian, Development of an ultrahigh speed robot FALCON using wire drive system, IEEE Int. Conf. Rob. Automat, 1995, 215-220.

[5] A.J. Madhani, G. Niemeyer, J.K. Salisbury, The black falcon: a teleoperated surgical instrument for minimally invasive surgery, in: IEEE/RSJ Int. Conf. Intell. Robots Syst., vol. 2, 1998, pp. 936-944.

[6] S. Hirose, Biologically Inspired Robots, Oxford University Press, Oxford, Great Britain, 1993.

[7] A. Morecki, Z. Busko, H. Gasztold, K. Jaworek, Synthesis and control of the anthropomorphic two-handed manipulator, in: Proc. 10th Int. Symp. Ind. Robots, 1980, pp. 461-474.

[8] L.W. Tsai, J.J. Lee, Kinematic analysis of tendon-driven robotic mechanisms using graph theory, ASME J. Mech., Trans., Automat. Des. 111 (1) (1989) 59-65. 
[9] M.G. Hollars, R.H. Cannon, Initial experiments on the end-point control of a two-link manipulator with flexible tendons, ASME Winter Annual Meeting, Miami, FL, 1985.

[10] G.M. Prisco, M. Bergamasco, Dynamic modeling of a class of tendon-driven manipulators, in: Proc IEEE Int. Conf. Rob. Automat., Monterey, California, 1997.

[11] Y.H. Lee, J.J. Lee, Modeling of the dynamics of tendon-driven robotic mechanisms with flexible tendons, Mech. Mach. Theory 38 (12) (2003) 1431-1447.

[12] C.R. Johnstun, C.C. Smith, Modeling and design of a mechanical tendon actuation systems, ASME J. Dyn. Syst., Measur., Control 114 (1992) 253.

[13] M. Kaneko, W. Paetsch, H. Tolle, Input-dependent stability of joint torque control of tendon-driven robot hands, IEEE Trans. Ind. Electron. 39 (2) (1992) 96-104.

[14] L.W. Tsai, Robot Analysis-the Mechanics of Serial and Parallel Manipulators, John Wiley and Sons, Inc., 1999.

[15] H.K. Khalil, Nonlinear Systems, second ed., Prentice-Hall, Inc., New Jersey, 1996. 